\title{
In Situ Transmission Electron Microscopy Study on the Deformation Responses of Additively Manufactured Multiphase Ni-based Superalloy
}

\author{
Qianying Guo, Michael M. Kirka and Kinga A. Unocic \\ Oak Ridge National Laboratory, Oak Ridge, Tennessee, United States
}

MAR-M247 is a Ni-based superalloy, which is widely used in gas turbine blade-based applications and in the aerospace industry due to its good mechanical properties, high creep strength, good castability, and an excellent oxidation resistance [1]. However, this alloy exhibits poor weldability owing to its high $\mathrm{Cr}$ and Co concentration, which are mainly attributed to partitioning in the $\gamma$ phase [2]. Recently, additive manufacturing (AM) processing efforts are focused on processing of MAR-M247 structures to solve and understand weldability issues. In this study, MAR-M247 alloy, with large cuboidal $\gamma^{\prime}$ phases and narrow $\gamma$ channels in elongated grains (texture) was prepared by electron beam melting (EBM). The variations in the $\gamma^{\prime}$ phases were observed throughout the build height $(\sim 138 \mathrm{~nm}$ at bottom of the build vs. $360 \mathrm{~nm}$ at the top of the build). The deformation responses of a single $\gamma^{\prime}$ phase and multiphase $\left(\gamma^{\prime} / \gamma / \gamma^{\prime}\right)$ were analyzed using an in situ transmission electron microscopy (TEM) deformation holder. Scanning transmission electron microscopy (STEM) with energy dispersive X-ray spectroscopy (EDS) were utilized to identify the different phases in the EBM MAR-M247 alloy in order to correlate this with the nanoscale deformation behavior.

The site-specific-lamella for nanopillars were fabricated by using focus ion beam (FIB) in situ lift-out technique approximately from the [102] orientation. A final annular top-down milling process was performed to prepare the nanopillars from the lamella. The nanopillars were then deformed by a flat punch indenter in a Bruker PI95 pico-indenter in situ TEM holder and within a Hitachi HF-3300 (S)TEM operated at $300 \mathrm{kV}$. The in situ tests were displacement controlled, with the speed at $1 \mathrm{~nm} / \mathrm{s}$ and the maximum displacement of $100 \mathrm{~nm}$. The tests were manually stopped when fracture/failure occurred.

The microstructure evolution of the nanopillars during in situ compression tests were studied by bright field (BF) TEM imaging. Figure 1 (a)-(c) shows BF-TEM images of pre-deformed, during deformation and post-deformed nanopillar. An inclined fracture was noted in the middle of this nanopillar. A further chemical analysis was performed on this deformed nanopillar using a FEI F200X Talos operating at 200 $\mathrm{kV}$ with Super-X EDS detector. A sandwich of $\gamma^{\prime} / \gamma / \gamma^{\prime}$ phase structure was identified in this nanopillar, based on the Al partitioning in $\gamma^{\prime}$ phase (Fig. 1e) and Co and Cr partitioning in $\gamma$ phase (Fig. 1f-g) [2]. The deformation initiated at the side of the $\gamma^{\prime}$ phase (arrow) that was further away from the indenter/nanopillar interface, then propagated across $\gamma$ phase and reached the bottom of the second $\gamma^{\prime}$ phase. This caused an early failure of the nanopillar at $\sim 80 \mathrm{~nm}$, or at a strain of 0.2 (Fig. 2). Another nanopillar was also in situ deformed and its microstructure evolution is shown in Figure $1(\mathrm{~h})-(\mathrm{j})$. This nanopillar mainly contained $\gamma^{\prime}$ phase with the bottom being $\gamma$ phase (called $\gamma / \gamma^{\prime}$ nanopillar). There was no fracture during deformation up to the displacement of $100 \mathrm{~nm}$. Chemical analysis confirmed that this nanopillar was mainly $\gamma^{\prime}$ phase based (with a limited $\gamma$ phase existed at the very bottom of this nanopillar). Without the interferences from the $\gamma$ phase and related $\gamma / \gamma^{\prime}$ interfaces, the strain of the single phase $\gamma^{\prime}$ nanopillar can be as much as 0.26 (30\% higher than the multiphase nanopillar), while maintain the similar stress level (Fig. 2). For a comparison a tensile stress-strain curve of a bulk casting MAR-M247 was also included in Figure 2. As expected, higher ultimate stress was observed but much lower ultimate strain comparing to the results from testing of the nanopillars. Such differences are related to the volume of the deformed specimen. The exceptional mechanical performance of AM MAR-M247 depends on volume fraction and morphology of 
the $\gamma^{\prime}$ phase. The current study shows that in situ TEM deformation analysis is reliable technique to study local deformation responses of this alloy with or without multiple phases at nanoscale [3].
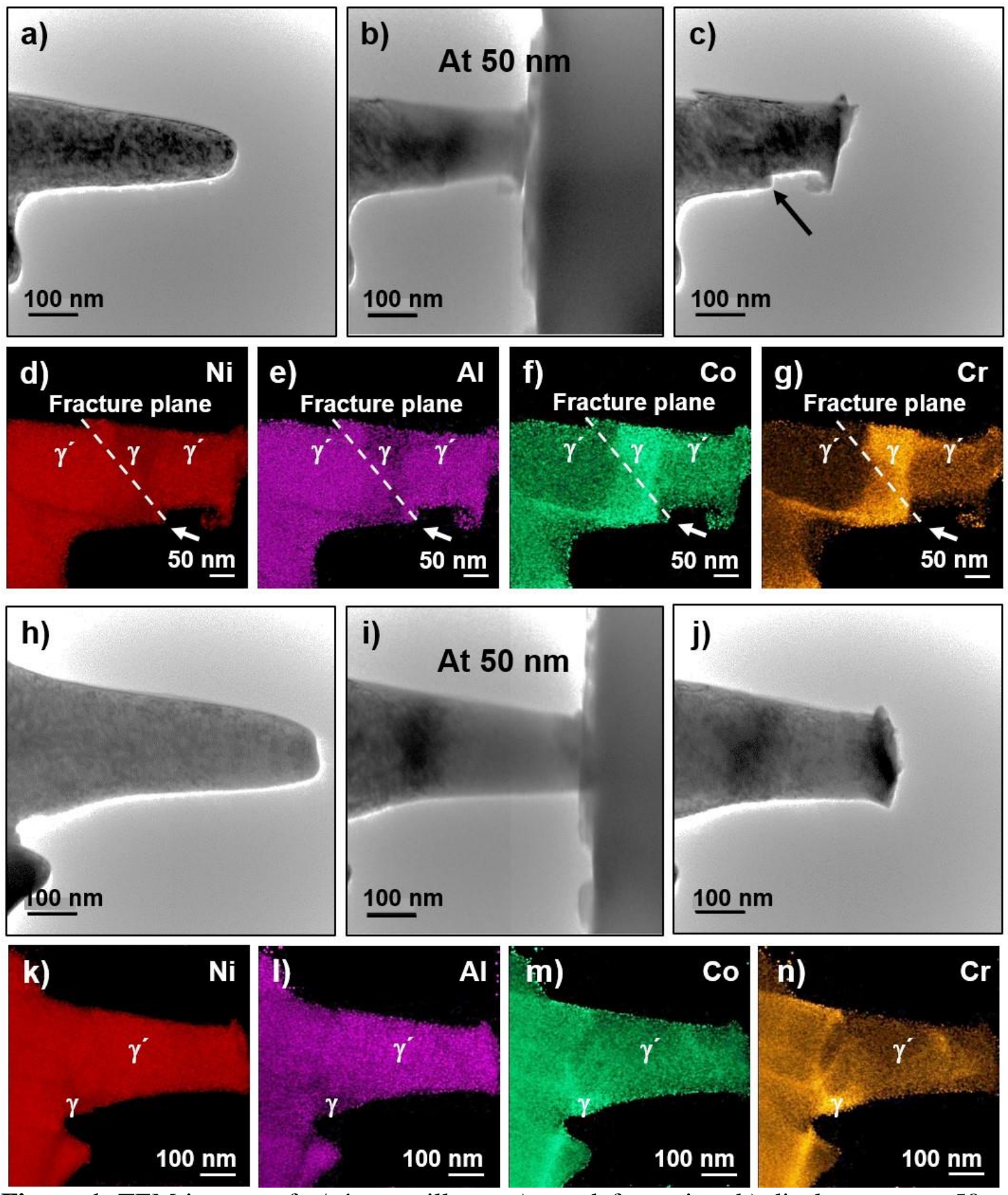

Figure 1. TEM images of $\mathrm{g} / \mathrm{g}^{\prime}$ nanopillar at a) pre-deformation, b) displacement at $50 \mathrm{~nm}$, and c) postdeformation conditions. Correlated quantified $\mathrm{Ni}, \mathrm{Al}, \mathrm{Co}$, and Co elemental maps of the tested $\mathrm{g}^{\prime} / \mathrm{g} / \mathrm{g}^{\prime}$ nanopillar are shown in (d), (e), (f), and (g). TEM images of the second g/g' nanopillar at $h$ ) predeformation, i) displacement at $50 \mathrm{~nm}$, and j) post-deformation conditions. Correlated quantified $\mathrm{Ni}, \mathrm{Al}$, Co, and Co elemental maps of the tested g nanopillar are shown in (k), (l), (m), and (n). 


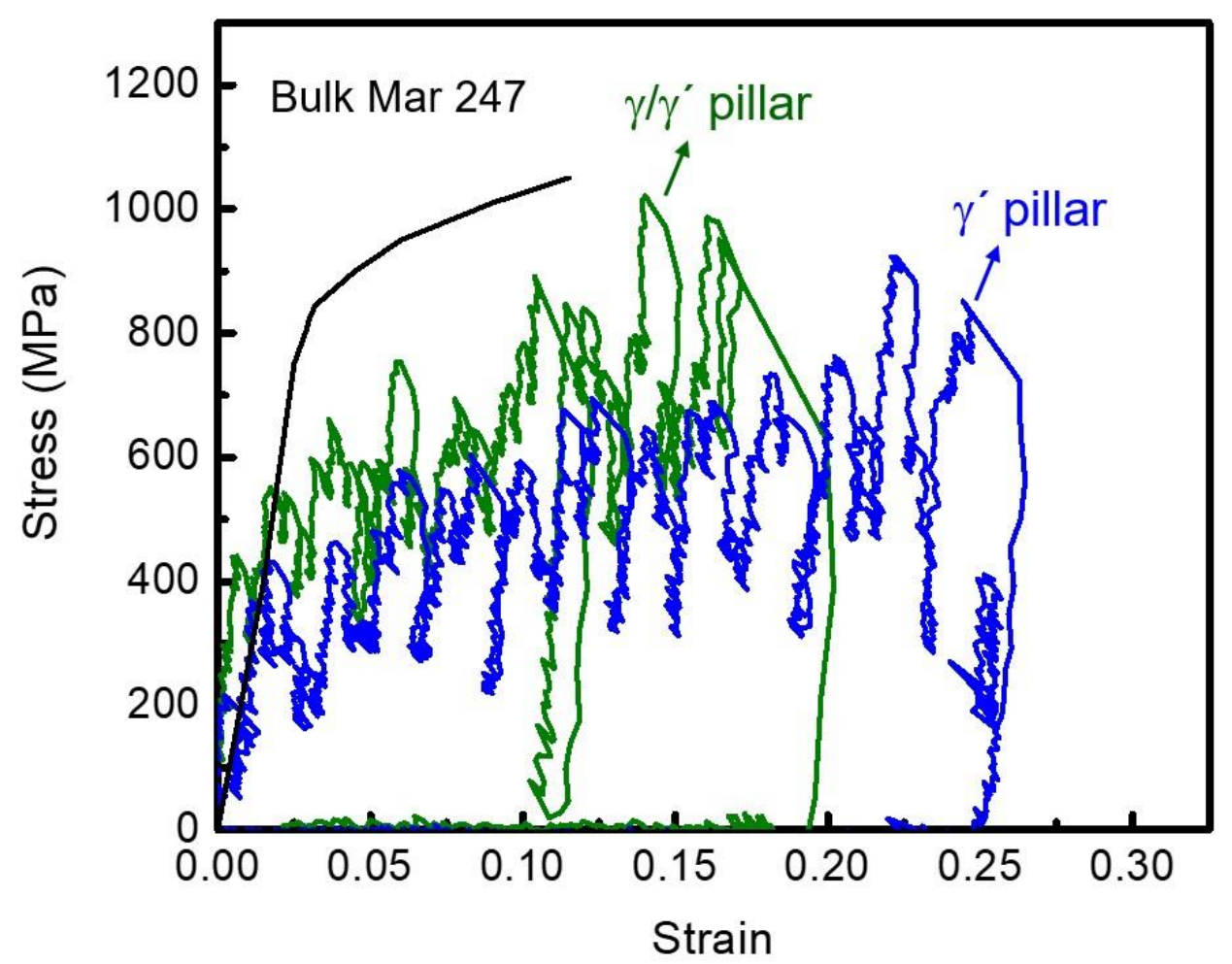

Figure 2. Stress-strain curves of both nanopillars extracted from EBM MAR-M247. A bulk MAR-M247 curve is added for comparison.

\section{References}

[1] M. Kaufman, Superalloys (1984), p. 43.

[2] Z. Mao, et al., Acta Mat. 166 (2019), p. 702.

[3] Sample was provided by the Manufacturing Demonstration Facility, which was sponsored by DOE's Office of Energy Efficiency and Renewable Energy. Microscopy conducted using instruments that are part of ORNL Nuclear Science User Facilities, which is a U.S. DOE Office of Science User Facility. 